Ana Zwitter Vitez

Fakulteta za humanistične študije, Univerza na Primorskem

Filozofska fakulteta, Univerza v Ljubljani

Ana.zwitter@guest.arnes.si
$81 ' 322.5:[811.133 .1+811.163 .6]$

DOI: $10.4312 /$ vestnik.6.63-77

\title{
LES MOYENS STRATÉGIQUES POUR PRENDRE LA PAROLE EN FRANÇAIS ET EN SLOVÈNE
}

\section{INTRODUCTION}

Dans la conversation quotidienne, il arrive souvent que deux locuteurs veuillent prendre la parole au même moment. Il s'agit d'un phénomène si fréquent qu'on ne se rend même pas compte des moyens utilisés dans cette lutte pour la prise de la parole. Sans investissement réflexif, les interlocuteurs se servent de diverses structures linguistiques combinées de manière très complexe afin d'atteindre leurs buts communicatifs. S'il paraît assez facile de dire si un locuteur a été efficace ou non dans la prise de la parole, la tâche d'identifier les moyens stratégiques est plutôt complexe.

Le défi principal de la contribution sera de répondre à la question suivante : pourquoi, dans un échange oral spontané, lorsque deux locuteurs parlent en même temps, l'un garde la parole tandis que l'autre l'abandonne ? Nous allons essayer de répondre à cette question à l'aide des analyses antérieures réalisées sur le français et d'une analyse détaillée de conversations orales spontanées en slovène.

\section{CADRE THÉORIQUE}

Si la production orale se retrouve exclue du courant structuraliste qui la considère comme un imbroglio impropre à toute analyse rigoureuse (Saussure 1916), elle attire un intérêt croissant ces dernières décennies. Ce succès est probablement dû à la grande valeur applicative des analyses du discours oral dans les domaines de l'enseignement, des technologies du langage et de la traduction.

Plusieurs théories et approches d'analyse de l'oral ont été élaborées sur les fondements posés par le schéma classique de la communication (Jakobson 1963). Ainsi, Émile Benveniste formule la théorie de l'énonciation (1974: 80): "L'énonciation est cette mise en fonctionnement de la langue par un acte individuel d'utilisation. " La linguistique de 
l'énonciation aborde les traces que le sujet parlant laisse dans son discours et analyse les éléments concrets de la situation de l'énonciation : le locuteur, l'interlocuteur, le lieu et le moment de leur échange.

Le discours oral est exploré aussi par l'approche pragmatique (Moeschler 1985) qui se propose non pas d'analyser la signification de la proposition, mais la fonction de l'acte de langage réalisé par l'énoncé. L'approche conversationnelle (Sacks 1970) analyse à partir d'enregistrements audio et vidéo la structure et les mécanismes principaux qui gèrent l'interaction verbale et l'alternance des tours de parole. L'approche macrosyntaxique met les données prosodiques en relation avec des unités de macro-syntaxe, parce que la syntaxe de la phrase et des propositions «ne suffit pas à rendre compte de certaines organisations de la langue parlée » (Blanche Benveniste 1997).

Les recherches sur le slovène oral portent sur des structures différentes : le discours quotidien (Toporišič 1970), l'intonation phrastique (Vitez 1995), les connecteurs discursifs (Schlamberger Brezar 2000), les genres discursifs (Smolej 2006), l'acquisition du langage (Kranjc 1997) et les éléments de spontanéité en fonction des technologies du langage (Verdonik 2007).

Les théories et les recherches présentées ont tour à tour contribué à l'élaboration de cette étude. D'abord, elles ont indiqué les pistes de recherche qu'il faut encore réaliser et, ensuite, une fois l'objet d'analyse déterminé, elles ont facilité le choix d'une méthodologie optimale.

\subsection{La théorie du paragraphe oral}

La théorie sur laquelle repose le cadre méthodologique de la présente étude est fondée par Mary-Annick Morel et Laurent Danon-Boileau et publiée dans leur ouvrage Grammaire de l'Intonation (1998). Elle est issue de l'approche énonciative d'Émile Benveniste et de son idée que tout sujet parlant laisse dans son discours des traces de sa subjectivité. Les auteurs partent du postulat que, dans l'échange oral spontané, l'intonation représente un moyen stratégique important qui joue un rôle crucial dans la construction d'unités cohérentes du discours.

Dans la méthodologie de Morel et de Danon Boileau (1998), l'intonation englobe quatre indices suprasegmentaux : la fréquence fondamentale (F0), l'intensité, la durée et les pauses. Chacun d'entre eux se voit accorder une valeur iconique de base. La valeur de la hauteur mélodique permet d'attirer l'attention sur un fragment du discours, l'intensité indique la façon dont le locuteur gère son tour de parole, La durée relative d'une syllabe indique l'état de la formulation des idées que le locuteur s'apprête à exposer, et la pause silencieuse indique le tournant au sein d'un cadre déjà constitué.

Selon la méthodologie de Morel et de Danon Boileau (1998), la prise en compte du droit à la parole et l'anticipation d'une revendication de la parole sont donc liées au rôle 
stratégique de l'intensité. Les variations de l'intensité indiquent l'attitude du locuteur par rapport à la prise de la parole :

- $\quad$ si le locuteur se situe dans la continuité, il assure son droit à la parole en maintenant une intensité constante,

- s'il anticipe une prise de parole imminente du colocuteur et qu'il veut manifester qu'il tient à conserver son tour de parole, il va donner plus de force à l'intensité,

- $\quad$ s'il se prépare à céder son droit à la parole, par exemple à la fin d'une question totale, ou s'il n'a aucune appréhension à se le voir revendiquer, il va laisser chuter l'intensité.

Comme dans un échange oral spontané les indices intonatifs opèrent en interaction mutuelle, il faut prendre en considération la conjoncture régulière de plusieurs indices : quand le locuteur veut attirer l'attention de l'autre et en même temps garder la parole, il va monter simultanément la fréquence fondamentale et l'intensité. En revanche, quand il ne veut plus garder l'attention de celui qui l'écoute ni garder le droit de la parole, il va baisser simultanément les deux indices, le F0 et l'intensité. Cette chute conjointe et rapide de l'intensité et de F0 représente l'indice fiable de la fin de l'unité de l'oral, appelée le paragraphe oral.

Si le paragraphe oral et sa cohésion intérieure peuvent être délimités par les indices prosodiques, les constituants qui le composent sont identifiables par les critères morphosyntaxiques et discursifs. Un paragraphe comprend au moins un rhème (elle est décapotable), précédé d'un ou de plusieurs préambules qui sont des segments à valeur thématique et modale (mais c'est bon). Le rhème peut, sous certaines conditions, être suivi d'un postrhème (exemple (1)) :

mais c'est bon / elle est décapotable / la bagnole

(Morel, Danon Boileau 1998 : 22)

La structure type du paragraphe oral en français est résumée ci-dessous :

\section{paragraphe oral type $=$ préambule + rhème + postrhème.}

Le préambule constitue l'élément initial du paragraphe oral. En français, le préambule est extrêmement décondensé, formé de plusieurs éléments juxtaposés sans marque de fonction syntaxique. Le premier élément du préambule est le ligateur qui précise le lien de ce qui va se dire avec ce qui a déjà été dit ; suivent les indices de modalité exprimant le point de vue et donnent à ce qui va se dire une valeur épistémique ou appréciative, le cadre délimitant une zone de sens et le support lexical disjoint qui place un objet de discours dans cette zone. 
Les constituants du préambule se suivent dans l'ordre suivant :

\section{préambule $=$ ligateur + point de vue + modus dissocié + cadre + support lexical disjoint}

Dans le discours oral spontané, il arrive rarement que tous les constituants soient présents dans un seul paragraphe, il peut y en avoir un ou deux seulement (exemple (2):

(2)

préambule

ligateur modus cadre cadre sld

parce que c'est vrai qu' souvent dans notre courrier on a des gens

rhm

qui disent ah oui je suis à la retraite je ne sais pas quoi faire je m'ennuie je m'embête (Morel, Danon Boileau 1998 : 24)

\section{CADRE MÉTHODOLOGIQUE}

Tout en suivant le schéma théorique de Morel et de Danon Boileau (1998), nous avons essayé d'identifier l'ensemble des moyens linguistiques les plus efficaces lors de la lutte pour la parole. Pour ce faire, nous avons examiné en détail trois conversations orales spontanées en slovène aux niveaux prosodique, morphosyntaxique et discursif en observant ensuite l'effet des structures utilisées dans la suite de l'échange oral.

\subsection{Corpus}

Le corpus comporte trois conversations réalisées par six locuteurs qui se connaissent très bien entre eux. Chaque conversation consiste des locuteurs représentant une classe d'âge : de 20 à 34 ans, de 35 à 59 ans, de 60 à 80 ans. Afin d'atteindre le degré de spontanéité optimal, nous avons d'abord installé les microphones et les caméras, ensuite nous avons demandé aux locuteurs de patienter quelques minutes pour des raisons techniques. Pendant qu'ils «attendaient» le début de l'enregistrement, ils ont tous entamé une conversation sans savoir qu'ils étaient déjà enregistrés.

Le tableau (1) représente les données quantitatives des quatre conversations analysées : la longueur (en secondes), le nombre de mots produits par chacun des locuteurs et le nombre total de mots produits dans chaque conversation. 
Tableau (1): Données quantitatives du corpus.

\begin{tabular}{|l|c|l|c|c|}
\hline Conversation & Duree $(\mathbf{s e c})$ & Locuteur & Nb de mots/loc. & $\boldsymbol{\Sigma}$ Nb mots \\
\hline $\mathbf{1 M o n}$ & 81 & A & 223 & 223 \\
\hline $\mathbf{1}$ & 115 & A & 106 & 427 \\
\hline & & B & 321 & \\
\hline $\mathbf{2}$ & 225 & C & 621 & 902 \\
\hline & & D & 281 & \\
\hline $\mathbf{3}$ & 178 & E & 310 & 574 \\
\hline & & F & 264 & \\
\hline
\end{tabular}

\subsection{Transcription et préparation des données}

Les segments analysés ont été transcrits à l'aide du logiciel Praat (Figure (1)) qui permet de visualiser la transcription et les courbes prosodiques indiquant l'intensité, la hauteur mélodique et la durée des segments transcrits.

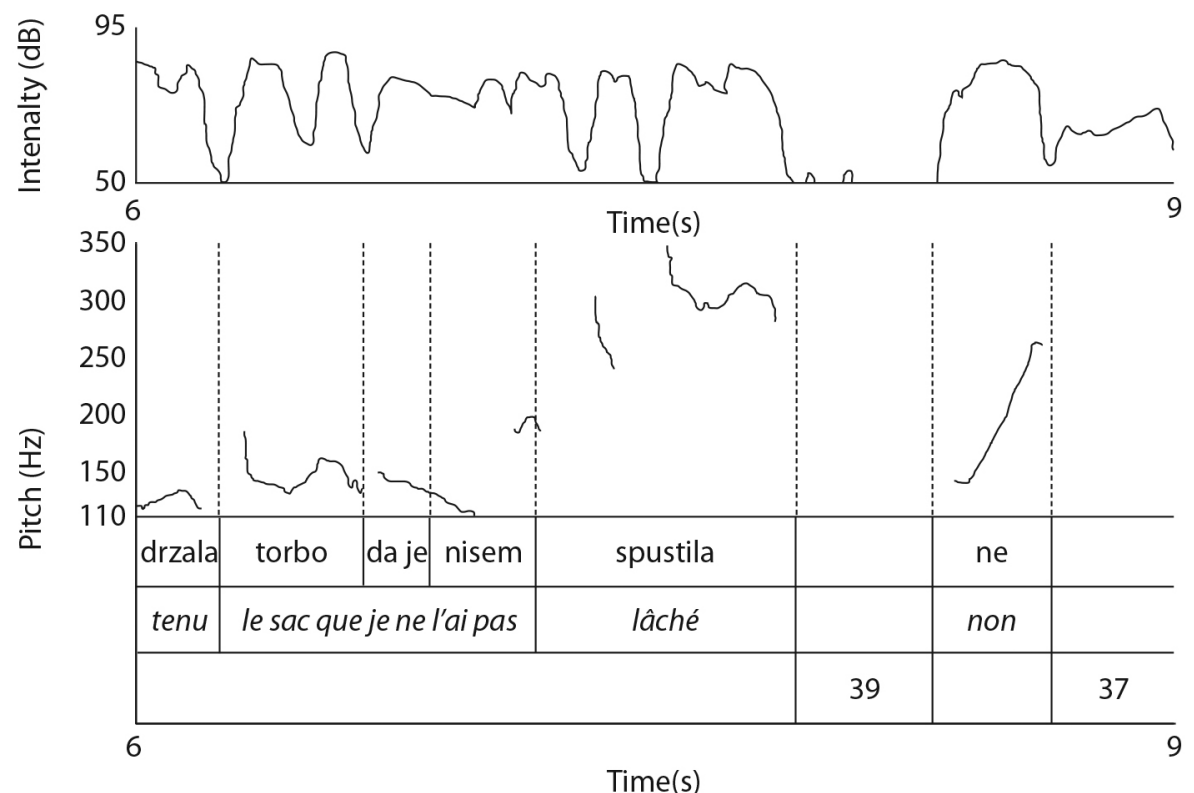

Figure (1): Transcription dans le logiciel Praat.

En vue d'une lecture simplifiée, nous avons transporté les unités de mesure absolues ( $\mathrm{dB}$ pour l'intensité et $\mathrm{Hz}$ pour la hauteur mélodique) en niveaux relatifs d'intensité (Tableau (2)) et de hauteur mélodique (Tableau (3)). 
Tableau (2): Les degrés relatifs de l'intensité.

\begin{tabular}{|l|l|l|l|l|}
\hline Convers. & Locuteur & Forte & Moyenne & Basse \\
\hline $\mathbf{1}$ mon & A & $50-63$ & $64-76$ & $77-90$ \\
\hline $\mathbf{1}$ & A & $50-63$ & $64-76$ & $77-90$ \\
\hline & B & $60-71$ & $72-83$ & $84-95$ \\
\hline $\mathbf{2}$ & C & $45-58$ & $59-71$ & $72-85$ \\
\hline & D & $40-53$ & $54-66$ & $67-80$ \\
\hline $\mathbf{3}$ & E & $40 \vee 53$ & $55-66$ & $68-80$ \\
\hline & F & $35-48$ & $49-61$ & $50-75$ \\
\hline
\end{tabular}

Tableau (3) : Les niveaux relatifs de la bauteur mélodique.

\begin{tabular}{|l|l|l|l|l|l|}
\hline Convers. & Locuteur & Niveau H1 & Niveau H2 & Niveau H3 & Niveau H4 \\
\hline $\mathbf{1}$ Mon & A & $110-170$ & $171-230$ & $231-290$ & $291-350$ \\
\hline $\mathbf{1}$ & A & $90-137$ & $138-185$ & $186-232$ & $233-280$ \\
\hline & B & $140-195$ & $196-250$ & $251-305$ & $306-360$ \\
\hline $\mathbf{2}$ & C & $90-115$ & $116-140$ & $141-165$ & $166-190$ \\
\hline $\mathbf{3}$ & D & $110-152$ & $153-194$ & $195-236$ & $237-280$ \\
\hline & E & $70-100$ & $101-130$ & $131-160$ & $161-190$ \\
\hline & F & $60-94$ & $95-128$ & $129-162$ & $163-195$ \\
\hline
\end{tabular}

\subsection{Conventions de transcription}

Les enregistrements sont transcrits à l'aide de principes de transcription orthographique adaptée à l'image acoustique du mot. Elle comporte les marques suivantes :

$\S$ : $\quad$ chevauchement de paroles

$\S \S$ : $\quad$ fin de chevauchement de paroles

\{\} : pause

(h): pause remplie par une inspiration audible

$\backslash$ : rupture dans la construction syntaxique

: : allongement d'un son

MAJ : prononciation appuyée ; accent d'insistance

$<\mathrm{r}>$ : rire

\section{ANALYSE}

L'analyse a été effectuée aux niveaux prosodique, morphosyntaxique et discursif. 


\subsection{Prosodie}

D'abord, nous avons comparé la forme prosodique de la production des six locuteurs au moment où ils s'efforcent, chacun à son tour, de reprendre la parole à l'interlocuteur (Figures 2, 3 et 4).

Figure 2 : Prosodie dans la conversation 1.

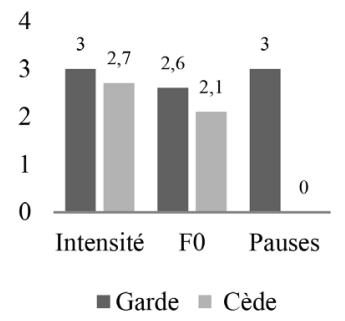

Figure 3 : Prosodie dans la conversation 2.

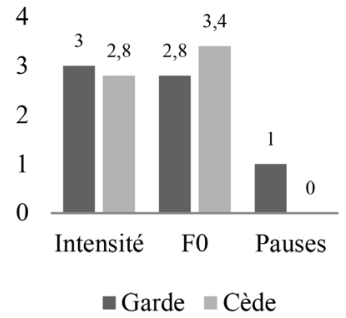

Figure 4 : Prosodie dans la conversation 3.

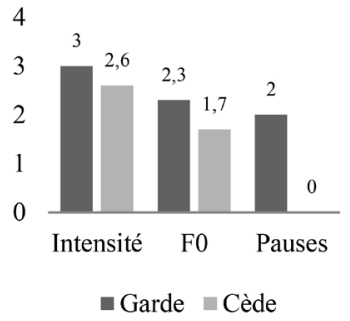

Les résultats montrent que, dans les trois conversations, le locuteur qui garde la parole fait monter l'intensité au niveau très élevé (le niveau 4 dans les trois situations). Pourtant, le locuteur qui cède la parole semble, lui aussi, hausser l'intensité à un niveau assez élevé (3,7 dans la conversation 1, 3,8 dans la conversation 2 et 3,6 dans la conversation 3).

Au niveau des pauses, les locuteurs qui gardent la parole font tout compris 6 pauses tandis que ceux qui la cèdent n'en font aucune. L'exemple (3) montre la fonction de la pause dans l'annulation de ce qui a déjà été dit :

(3)

\section{LOC C}

sej je bil šter leta z njo al kok ne \{\}

en tout cas il est resté quatre ans avec elle ou combien hein \{\}

pa to§pod težkimi pogoji ne§§

et ça §dans des conditions difficiles hein§§

LOC D

$\S$ ni to $\backslash\{\}$ mislm \{\}$\S \S$ ni to prov

§ce n'est pas| \{\} je veux dire \{\}$\S \S$ ce n'est pas bien

da da \{\} a veš

que que \{\} tu sais

L'exemple (1) montre que la locutrice $\mathrm{D}$ abandonne la construction syntaxique $n i$ to (ce n'est pas ), fait une pause et repart de nouveau par le ligateur mislm (je veux dire), fait encore une pause et recommence par une intensité très forte la construction ni to prov da da \{\} a veš (ce n'est pas bien que que \{\} tu sais), et le locuteur $\mathrm{C}$ cède la parole. 
En ce qui concerne les moyens prosodiques, nous pouvons conclure que les locuteurs qui produisent des énoncés avec une intensité très forte et annulent les opérations précédentes par les pauses semblent être très efficaces quand ils veulent garder ou prendre la parole à leur interlocuteur.

\subsection{Morphosyntaxe}

Le deuxième niveau d'analyse est représenté par les structures morphosyntaxiques. Cette catégorie nécessite une explication plus détaillée parce que l'analyse du discours enregistré a changé d'une manière radicale les critères d'analyse prévus. En effet, l'unité d'analyse de l'oral et ses sous-parties étant tellement différentes de la syntaxe de l'écrit, les catégories morphosyntaxiques traditionnelles n'ont pas beaucoup d'importance lorsqu'on veut analyser l'efficacité des locuteurs essayant de prendre ou de garder la parole.

Au contraire, il semble que les moyens les plus efficaces dans la lutte pour la parole soient représentés par des éléments directement issus du phénomène de la production spontanée de l'oral où le locuteur n'a pas le temps de planifier consciemment sa production verbale. Ces phénomènes sont regroupés en trois catégories :

- principes paradigmatiques sur l'axe syntagmatique (répétition, autocorrection, construction abandonnée),

- spécificités sur l'axe syntagmatique (ordre de mots changé, présence de pronom personnel avant la forme verbale, actualisateurs textuels comme ta (ce), un (ce), en (un), adresse directe de l'interlocuteur) et

- tournures au niveau textuel (questions imprévisibles).

Les Figures 5, 6, et 7 montrent les structures de spontanéité repérées lors de la lutte pour la parole.

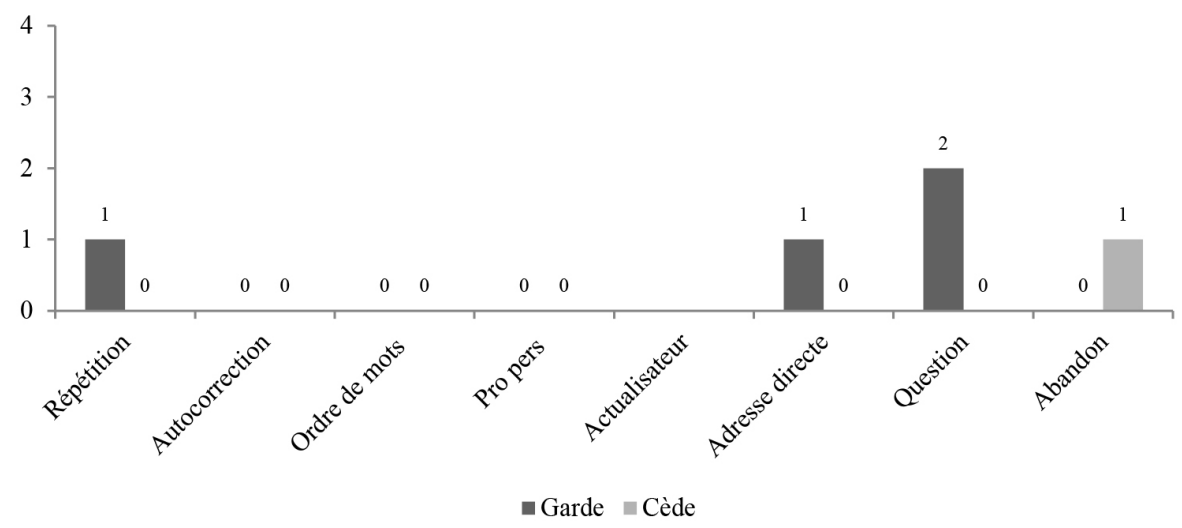

Figure 5 : Spécificités morphosyntaxiques dans la conversation 1. 


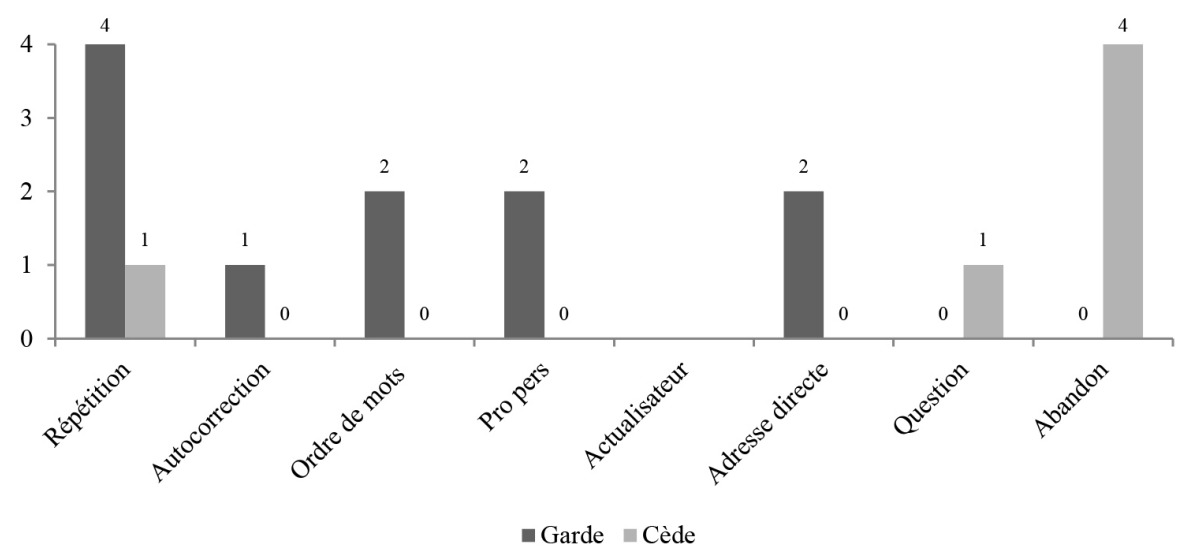

Figure 6 : Spécificités morphosyntaxiques dans la conversation 2.

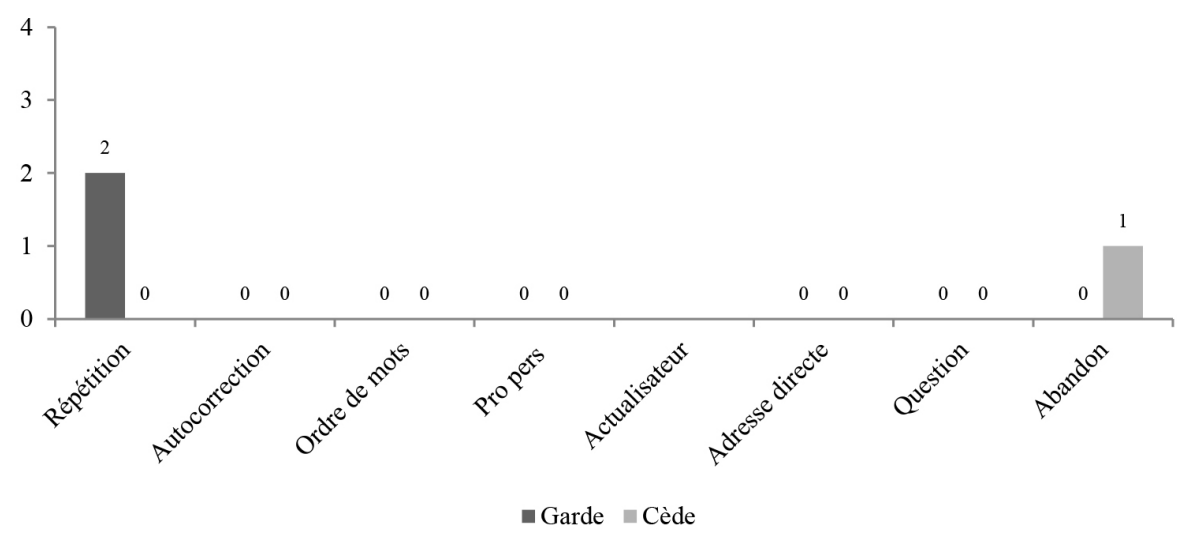

Figure 7 : Spécificités morphosyntaxiques dans la conversation 3.

Dans les trois conversations, les participants emploient des structures qui divergent de la langue standard. Il semble que les locuteurs qui gardent la parole ont tendance à produire plus de répétitions et d'autocorrections, ce qu'illustre l'exemple (4) :

(4)

\section{LOC D}

a razumeš $\S s i\{\}$ skenslaš enga in pol $\backslash \S \S$

tu comprends §tu te débarasses de qqn et puis $\mid \oint \oint$

LOC C

§pa sej to ni pa sej kaj pa je to slabga§§

§mais en tout cas ce n'est pas mais en tout cas qu'est-ce qu'il y a de mal\$§ 
Le locuteur C répète d'abord une partie de la structure pa sej to ni (mais en tout cas ce n'est pas) par pa sej (mais en tout cas), ensuite, il démarre de nouveau par la question kaj pa je to slabga (qu'est-ce qu'il y a de mal). La locutrice D, à son tour, abandonne la parole. Le phénomène de construction abandonnée est donc prévisiblement présent chez les locuteurs qui cèdent la parole.

Le deuxième phénomène remarqué, l'altération de l'ordre des mots, semble diverger aussi de descriptions morphosyntaxiques traditionnelles. Si l'ordre de mots le plus fréquent en slovène est sujet + verbe + complément, il apparaît que, dans la situation de la lutte pour la parole, certains locuteurs utilisent un ordre de mots atypique (exemple (5)) :

(5)

\section{LOC C}

sej je bil šter leta $\mathrm{z}$ njo al kok ne \{\}

en tout cas il est resté avec elle quatre ans ou combien hein \{\}

\section{pa toßpod težkimi pogoji ne§§}

et ça §dans des conditions difficiles hein§§

LOC D

\section{$\S$ ni to $\backslash\{\}$ mislm \{\}$\S \S$ ni to prov}

§ce n'est pas| \{\} je veux dire \{\}\&\& ce n'est pas bien

da da \{\} a veš

que que \{\} tu sais

L'exemple ci-dessus montre que la locutrice $\mathrm{D}$, qui garde la parole, répète la construction ni to $\mid$ ni to prov (ce n'est pas $\mid$ ce n'est pas bien) et utilise l'ordre non typique pour la langue slovène (ni to au lieu de to ni). Il semble qu'en utilisant cette structure, la locutrice réussit à accentuer davantage la portée de la négation qu'elle produit.

L'utilisation du pronom personnel accompagnant la forme conjuguée du verbe semble avoir l'effet d'accentuer le rôle de l'énonciateur et de grader l'effet de ce qui est dit (exemple (6).

(6)

\section{$\cdots$}

\section{LOC C}

in to je $\S$ un \{\} ž u ro d $\ \S$

et c'est $\{$ cette \{\} fête del§§

\section{LOC D}

$\S j a$ jaz ne vem zdej kašn problem $\S$ točno ma on a veš a al on $\backslash\{\}$ §bon moi je ne sais pas là quel problèmeß§ il a lui exactement tu sais si lui \{\} 
L'adresse directe de l'interlocuteur semble elle aussi avoir pour effet l'attention de l'interlocuteur (exemple (7)) .

(7)

LOC C

$\S$ tko da $\S$

alors

LOC D

ampak §to gre $\S$ loh v nedogled uroš

mais ça peut aller à l'infini uroš

La dernière catégorie de structures orales spontanées, question interrompant la production orale du premier locuteur, est liée au moment inattendu de sa production dans l'échange oral (exemple (8)) :

(8)

\section{LOC B}

(h) je v bistvu lahko §vsak večer noter $\ \S$

(h) en fait tous les soirs il pouvait à l'intérieur

LOC A

(h) $\{$ ti \{\} ob kašnem času $\S \S$ je blo to pri vam vzeto ob kašnem času \{\}

(h) §écoute \{\} quand est-ce que§§ cela s'est passé chez vous quand \{\}

L'exemple montre la force de la question de la locutrice A accompagnée de l'adresse directe de l'interlocutrice qui surgit lors de sa narration fluide. La locutrice B abandonne la parole et continue la narration dans le rythme imposé par la question de son interlocutrice.

\section{DISCOURS}

Le cadre théorique (Morel, Danon Boileau 1998) a montré que le paragraphe oral est constitué de plusieurs sous-parties (préambule, rhème et postrhème en français). Comme le préambule représente l'élément typique de la production spontanée du discours oral, nous avons analysé la structure et la longueur du préambule. L'analyse a porté sur le nombre de constituants discursifs $(1,2,3$ ou 4 constituants) produits par le locuteur qui garde la parole et par celui qui la cède (Figures 8, 9 et 10). 
Figure 8: Préambule dans la conversation 1.

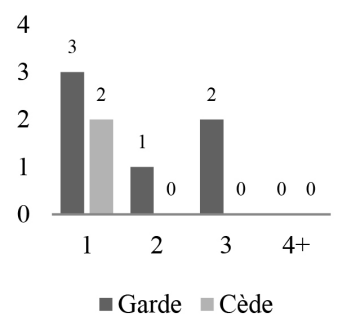

Figure 9: Préambule dans la conversation 2.

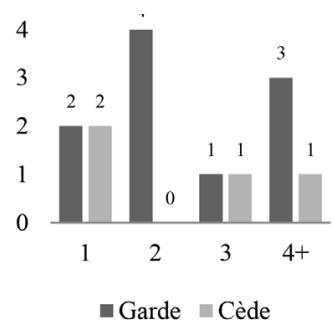

Figure 10: Préambule dans la conversation 3.

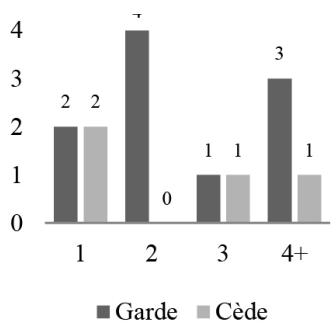

Les locuteurs qui gardent et qui cèdent la parole produisent un nombre plus ou moins équivalent de préambules courts (à un constituant). La différence devient apparente à partir des préambules à 2, 3 ou 4 constituants qui sont plus fréquemment utilisés par les locuteurs qui gardent la parole. L'exemple (9) illustre le rapport entre la longueur du préambule et l'efficacité du locuteur :

(9)

\section{LOC D}

jaz mislm $\S$ da to ni prov da morš bit $\ \S$

moi je pense §que ce n'est pas bien qu'il faut êtrel§§

\section{LOC C}

$\S$ okej mogoče zdej v tem primeru§§\{\} mogoče v primeru jerneja \{\}

§ok peut être là dans ce cas-làßई \{\} peut-être dans le cas de jernej \{\}

La locutrice D introduit son discours par un point de vue et un modus jaz mislm (moi je pense) et continue son énonciation par le rhème da to ni prov (que ce n'est pas bien), où intervient le locuteur $\mathrm{C}$ par un ligateur okej (ok), un modus mogoče (peut-être), un nouveau ligateur zdej (maintenant) et un cadre $v$ tem primeru (dans ce cas-là), ce qui suffit pour que son interlocutrice abandonne la parole.

Ces résultats mènent à la conclusion que les locuteurs produisant plus d'éléments de préambule semblent garder la parole plus fréquemment que ceux qui formulent des préambules courts.

\section{SYNTHÈSE}

Les combinaisons les plus fréquentes de moyens stratégiques utilisés lorsque les deux locuteurs veulent prendre la parole au même moment sont condensées par le tableau (4) et englobent les niveaux prosodique, morphosyntaxique et discursif. 
Tableau (4) : Structures stratégiques pour garder et pour céder la parole.

\begin{tabular}{|l|l|l|}
\cline { 2 - 3 } \multicolumn{1}{c|}{} & Locuteur qui garde la parole & Locuteur qui cède la parole \\
\hline Prosodie & nombreuses pauses & pauses peu nombreuses \\
\hline Msy & $\begin{array}{l}\text { répétition, autocorrection } \\
\text { ordre de mots changé } \\
\text { verbe précédé de pronom personnel } \\
\text { adresse directe de l'interlocuteur } \\
\text { question à un moment de conversation } \\
\text { imprévisible }\end{array}$ & \\
\hline Discours & préambule long (ligateur, modus, cadre) & préambule court \\
\hline
\end{tabular}

Le tableau (4) montre que les locuteurs qui ont gardé le droit à la parole ont produit un nombre important de pauses, de répétitions et d'autocorrections, ils ont souvent utilisé un ordre de mots atypique et le verbe précédé de pronom personnel pour accentuer l'importance de ce qu'ils disent. Leur production orale se caractérise par des préambules très longs. Les locuteurs qui ont cédé le droit à la parole ont produit peu de pauses et des préambules plutôt courts.

\section{CONCLUSION}

En vue de répondre à la question de savoir pourquoi, dans un échange oral, un locuteur prend la parole et l'autre l'abandonne, nous avons étudié les analyses réalisées sur le français et examiné trois conversations spontanées en slovène aux niveaux prosodique, morphosyntaxique et discursif.

Au niveau prosodique, l'analyse du corpus révèle les résultats prévus par les analyses du français : les locuteurs dont la production verbale est marquée par une forte intensité et par une fréquence importante de pauses vont, dans la plupart des cas, garder la parole. En ce qui concerne les structures morphosyntaxiques, des phénomènes comme la répétition, l'autocorrection et l'ordre de mots atypique se révèlent des moyens stratégiques efficaces pour garder ou prendre la parole. Au niveau discursif, les locuteurs qui produisent des préambules longs avec beaucoup de ligateurs, de modus et de cadres se révèlent plus efficaces que ceux qui en utilisent moins.

Les résultats de l'étude montrent que les structures traditionnellement traitées comme des faiblesses rhétoriques (intensité très forte, répétition, autocorrection, ordre de mots atypique, préambule long) semblent être très efficaces lorsque deux locuteurs veulent prendre la parole en même temps. Malgré tout, une piste de recherche reste ouverte; celle qui permettrait de préciser le rapport entre la capacité de garder la parole et l'impression laissée auprès de ses interlocuteurs. 


\section{RÉFÉRENCES}

BENVENISTE, Emile (1966) Problèmes de linguistique générale, tomes 1 et 2. Paris : Gallimard

BLANCHE-BENVENISTE, Claire (1991) Le français parlé. Études grammaticales, coll. Sciences du langage. Paris : Éditions du CNRS.

BLANCHE-BENVENISTE, Claire (1997) Approches de la langue parlée en français. Paris : Ophrys.

CULIOLI, Antoine (1999) Pour une linguistique de l'énonciation, Tome 3. Paris : Ophrys. JAKOBSON, Roman (1996) Lingvistični in drugi spisi. Ljubljana : Studia Humanitatis.

KERBRAT-ORECCHIONI, Catherine (1998) L'énonciation. De la subjectivité dans le langage. Paris : Armand Colin.

LACHERET-DUJOUR, Anne/Bernard VICTORRI (2002) La période intonative comme unité d'analyse pour l'étude du français parlé : modélisation prosodique et enjeux linguistiques. Verbum, 24/1-2, 55-73.

MOESCHLER, Jacques (1985) Argumentation et conversation. In : Eléments pour une analyse pragmatique du discours. Paris : Hatier.

MOREL, Mary Annick/Laurent DANON-BOILEAU (1998) Grammaire de l'intonation. Paris : Ophrys.

SACKS, Harvey (1995) Lectures on Conversation. Victoria : Blackwell Publishing.

SAUSSURE, Ferdinand de (1972) Cours de linguistique générale, édition critique préparée par Tullio De Mauro. Paris : Payot.

SCHLAMBERGER BREZAR, Mojca (2000) Les connecteurs en combinaison avec les marqueurs modaux : l'exemple du français et du slovène. Linguistica $\mathrm{XL} / 2$, Ljubljana, 273-282.

SMOLEJ, Mojca (2012) Besedilne vrste v spontanem govoru. Ljubljana : Znanstvena založba Filozofske Fakultete.

TOPORIŠIČ, Jože (1970) Slovenski pogovorni jezik. Slavistična revija 18, 55-70.

VERDONIK, Darinka (2007) Jezikovni elementi spontanosti v govoru. Maribor : Slavistično društvo Maribor.

VITEZ, Primož (1995) Analyse contrastive de l'intonation phrastique en français et en slovène. Linguistica XXXV, 2, Ljubljana, 257-274.

ZWITTER, Ana (2002) Les stratégies intonatives à l'échange oral en slovène et en français. Linguistica XLII, Ljubljana, 121-130. 


\section{RÉSUMÉ}

\section{Les moyens stratégiques pour prendre la parole en français et en slovène}

Le défi principal de la contribution est d'identifier les moyens stratégiques les plus efficaces lorsque deux locuteurs veulent prendre la parole au même moment. Nous avons examiné les études antérieures sur le français et analysé aux niveaux prosodique, morphosyntaxique et discursif trois conversations orales spontanées en slovène. Les résultats montrent que les locuteurs qui ont gardé leur droit à la parole produisent de nombreuses pauses, répétitions et autocorrections ainsi que des préambules plus longs que les locuteurs qui ont cédé leur droit à la parole.

Mots clés : oral spontané, prise de la parole, moyens stratégiques, pragmatique

\section{ABSTRACT}

\section{Strategic Structures for Turn-taking in French and Slovene}

The main challenge of the contribution was to identify the most effective strategic structures when two speakers want to speak at the same time. We reviewed state-of-the-art studies for French and analyzed three spontaneous conversations in Slovene from prosodic, morphosyntactic and discourse perspective. The results show that speakers who were efficient in taking their turn produced many pauses, repetitions and auto-corrections as well as longer discourse preambles than the speakers who were less successful in taking (or keeping) their turns.

Keywords: spontaneous speech, turn-taking, strategic structures, pragmatics

\section{POVZETEK}

\section{Strateška sredstva za prevzem besede $v$ francoščini in slovenščini}

V raziskavi smo želeli opredeliti najučinkovitejša strateška sredstva, ki jih uporabljajo govorci, ko se borijo za prevzem besede. Preučili smo sodobne študije govorjene francoščine in na prozodični, oblikoskladenjski in diskurzivni ravni analizirali tri spontane govorne izmenjave v slovenščini. Rezultati kažejo, da so govorci, ki so bili bolj učinkoviti pri prevzemanju besede, tvorili številne premore, ponavljanja in samopopravke, pa tudi bistveno daljše diskurzivne preambule kot govorci, ki so besedo prepustili.

Ključne besede: spontani govor, prevzemanje besede, strateška sredstva, pragmatika 